Andrzej Anderwald ${ }^{1}$

Uniwersytet Opolski, Polska

Wydział Teologiczny

\section{Zwischen Rom und Silicon Valley}

Papst Franziskus angesichts des technischen Fortschritts

In den Prozesseinreden gegen die Häretiker (De praescriptione haereticorum) fragte Tertullian $(\dagger 220)$ : „Was hat also Athen mit Jerusalem zu schaffen, was die Akademie mit der Kirche, was die Häretiker mit den Christen?" Diese Fragestellung hat die Wahl des Titels für die vorgeschlagenen Überlegungen inspiriert. Wenn man nämlich die Worte von Tertullian umschreibt, kann man fragen: Was hat Rom mit Silicon Valley zu tun? Das heißt: Was hat heute die Kirche mit der Wissenschaft und Technik zu tun? Hat sie überhaupt ein Interesse für eine Auseinandersetzung mit dem heutigen wissenschaftlich-technischen Fortschritt, für den Silicon Valley als Symbol gilt? Spielt die Kirche mit ihrem elaborierten Ausbildungssystem, mit ihren Werten und Traditionen eine Rolle für die Technik-Community? Welche Strategien schlägt die Kirche mit ihrem Ansatz für den technischen Fortschritt vor?

Die recht häufige Anerkennung der positiven Funktion des technologischen Fortschritts ist gleichzeitig eine Herausforderung für die humanistische Reflexion, die nicht nur über die Bedeutung der Produkte von Wissenschaft und Technik für die Menschheit, sondern auch über die eigentliche Idee des technologischen Fortschritts nachdenken soll. Diese Reflexion, die bereits auf philosophischer Ebene initiiert wurde, führte allmählich dazu, dass aus der allgemeinen philosophischen Reflexion eine neue spezifische Disziplin — die Philosophie der Technik — hervorging $^{2}$. Derzeit kommt auch eine weitere neue Forschungsperspektive — die theo-

${ }^{1}$ Kath. Priester, Dr. habil., ao. Univ.-Prof. Andrzej Anderwald — Lehrstuhlinhaber für Fundamentaltheologie und Religionswissenschaften an der Theologischen Fakultät der Universität Opole; e-mail: anderw@uni.opole.pl ORCID 0000-0001-5932-9845.

2 Siehe A. Nordmann, Technikphilosophie zur Einführung, Hamburg 2015; Ethisierung der Technik - Technisierung der Ethik. Der Ethik-Boom im Lichte der Wissenschafts- und Technik- 
logische - immer deutlicher zum Vorschein ${ }^{3}$. Es ist nicht zu übersehen, dass die Debatte über die Technik und den Fortschritt der Wissenschaften auch in den kirchlichen Verlautbarungen, wie z.B. den Enzykliken: Redemptor hominis (1978) von Johannes Paul II. (1920-2005) und Caritas in Veritate (2010) von Benedikt XVI. (geb. 1927) vorkommt. Auch Papst Franziskus (geb. 1936) greift das Thema auf. „Die Kirche verlangt nicht - schreibt Franziskus - den bewundernswerten Fortschrift der Wissenschaft aufzuhalten. Im Gegenteil, sie freut sich und findet sogar Gefallen daran, dass der Mensch die enorme Leistungsfähigkeit erkennt, die Gott dem menschlichen Geist verliehen hat ${ }^{\star 64}$. Die ständige Debatte über die Botschaft seiner Enzyklika Laudato si' (2015) im öffentlichen Raum ${ }^{5}$ ist für mich die Inspiration, um das oben formulierte Thema aufzugreifen. Unter Rom verstehe ich das Engagement der Kirche, besonders von Papst Franziskus, der hier eine starke Präsenz im Bereich der neuen Medien zeigt (z.B. auf Twitter oder auf Instagram) ${ }^{6}$. Obwohl die Umwelt- und Sozialfragen in den Diskussionen um diese Enzyklika dominieren, gibt es auch in ihr einige direkte Impulse zu einer Metareflexion über den wissenschaftlich-technischen Fortschritt. Wenn man auch die anderen Aussagen des Papstes über diese Problematik berücksichtigt, kann man mit Vorsicht von einem Vorschlag einer Theologie der Technik aus der Sicht von Franziskus sprechen. Das Ziel meiner Untersuchungen besteht darin, nach einer neuen Idee des technischen Fortschritts zu suchen, die dem Aufbau einer ganzheitlichen Vision der Wirklichkeit unter Achtung des höchsten Wertes der menschlichen Person ermöglicht. Um mit den Worten des Papstes das Ziel zu formulieren, kann man sagen: „Zugleich wird die dringende Notwendigkeit des Humanismus aktuell, der von sich aus die

forschung, hrsg. v. A. Bogner, Baden-Baden 2013.

${ }^{3}$ Siehe H.D. Mutschler, Die Gottmaschine. Das Schicksal Gottes im Zeitalter der Technik, Augsburg 1998, S. 243-248; S. Schleissing, Laien, Experten, Propheten: Zur Rolle der Theologen in Technikdiskursen [in:] Technik und Lebenswirklichkeit: Philosophische und theologische Deutungen der Technik im Zeitalter der Moderne, hrsg. v. A.M. Richter, Ch. Schwarke, Stuttgart 2014, S. 203-216.

${ }^{4}$ Franziskus, Apostolisches Schreiben Evangelii Gaudium (2013), Nr. 243, [online] http:// w2.vatican.va/content/francesco/de/apost_exhortations/documents/papa-francesco_esortazioneap_20131124_evangelii-gaudium.html [15.01.2019].

${ }_{5}^{5}$ Siehe z.B. die Internazionale Konferenzen im Zusammenhang mit dem Klima Gipfel in Katowice COP24: $W$ trosce o wspólny dom. Chrześcijanin na drogach ekologii (Katowice 30.11.2018); Ekologiczne przebudzenie: Misja Laudato si' — Chrześcijanie z klimatem (Katowice-Panewniki, 7-8.12.2019); Joint Symposium on Climate Change: Safeguarding Our Climate, Advancing Our Society (Katowice 10.12.2018); Siehe auch: O. Edenhofer, Klima, Kohle, Kapital. Zum Stand der internationalen Klimapolitik, „Stimmen der Zeit“ 143 (2018), H. 4, S. 263; A. Endras, Die Welt brennt, der Gipfel streitet, „Zeit Online”, [online] https://www.zeit.de/wirtschaft/2018-12/un-klimakonferenz-welt-klimagipfel-klimaschutz-kattowitz [5.01.2019].

${ }^{6}$ Die Twitter-Accounts von Papst Franziskus, die in neun Sprachen geführt werden, folgen über 46 Millionen Nutzer, davon über eine Million auf Polnisch — siehe: [online] https://misyjne. $\mathrm{pl}$ /franciszek-na-twitterze-drodzy-mlodzi-pomozcie-doroslym/ [12.01.2019]. 
verschiedenen Wissensgebiete [...] zusammenführt, um eine umfassendere wie integrierendere Perspektive zu erhalten" ${ }^{\text {"7 }}$.

Die Struktur der Überlegungen bezieht sich auf die Grundsätze der Soziallehre der sogenannten drei Schritte — die Grundsätze der Soziallehre, die in der Enzyklika Mater et Magistra (1961) von Papst Johannes XXIII. (1881-1963) beschrieben wurden:

Zunächst muß man den wahren Sachverhalt überhaupt richtig sehen; dann muß man diesen Sachverhalt anhand dieser Grundsätze gewissenhaft bewerten; schließlich muss man feststellen, was man tun kann und muß, um die überlieferten Formen nach Ort und Zeit anzuwenden. Diese drei Schritte lassen sich in den drei Worten ausdrücken: sehen, urteilen, handeln ${ }^{8}$.

Daher werden im Laufe der Überlegungen zunächst die Merkmale des technischen Fortschritts (1), dann dessen Bewertung (2) und schließlich ein ganzheitlicher Blick auf die Wirklichkeit (3) präsentiert.

\section{Technischer Fortschritt bzw. das technologische Paradigma}

Die Technologie und der damit verbundene wissenschaftlich-technischer Fortschritt sowie die wissenschaftlich-technische Zivilisation sind heutzutage die Größen, die alles oder fast alles in der umgebenden Wirklichkeit bestimmen. Die Produkte der Technik sind in allen Bereichen des menschlichen Lebens verbreitet. Es gibt keine Lebenssphäre (vom wirtschaftlichen Bereich bis zum Haushalt), die nicht unter ihrem Einfluss steht oder in der sie als überflüssig angesehen werden könnte. Das Konzept der modernen Technologie und des technischen Fortschritts wurde durch die wissenschaftlich-technische Revolution in der Wende vom 19. zum 20. Jahrhundert geprägt. In dieser Zeit wurde die Technik immer enger mit den mathematischen Naturwissenschaften verknüpft. Diese enge Verbindung führte zur Entstehung der qualitativ neuen Erfindungen, die die Verfügbarkeit von Reichtum deutlich erhöhten und damit einige Veränderungen in den sozialen Strukturen verursachten. Die moderne Technologie ist nicht nur ein Werkzeug oder eine Fähigkeit, wie die Etymologie des Wortes (Griechisch: techne - Kunst, Fähigkeit) zu suggerieren scheint, sondern sie ist durch eine gewisse Dynamik gekennzeichnet, die sich in einem kontinuierlichen, methodisch

${ }^{7}$ Franziskus, Enzyklika Laudato si', [online] https://www.katholisch.at/laudatosi, Nr. 141 [16.02.2019] (weiter wird mit „LS“ abgekürzt).

8 Johannes XXIII, Enzyklika Mater et Magistra (1961), [online] http://w2.vatican.va/content/ john-xxiii/de/encyclicals/documents/hf_j-xxiii_enc_15051961_mater.html, Nr. 236 [16.02.2019]. 
geplanten technischen Fortschritt ausdrückt. Man kann sagen, dass die moderne Technik nicht nur ihre Werke kennt und sie erschafft oder verarbeitet, sondern auch, wie Colin Miller feststellt, die Macht über sie hat und sie manipulieren $\mathrm{kann}^{9}$. Der gegenwärtige technologische Fortschritt zielt nicht nur darauf ab, die Zukunft zu entdecken oder zu beeinflussen, sondern sie auch mit der eigenen Methodik zu gestalten ${ }^{10}$.

Papst Franziskus verbindet noch deutlicher als seine zwei Vorgänger den technischen Fortschritt mit der Herrschaft des Menschen über die Natur bzw. die Herrschaft des Menschen über den Menschen oder einer Gesellschaft über eine andere. Eine solche Vorgehensweise führt zur Bildung eines spezifischen Paradigmas, nach dem ,eine Auffassung des Subjekts hervortritt, das im Verlauf des logisch-rationalen Prozesses das außen liegende Objekt allmählich umfasst und es so besitzt. Dieses Subjekt entfaltet sich, indem es die wissenschaftliche Methode mit ihren Versuchen aufstellt, die schon explizit eine Technik des Besitzens, des Beherrschens und des Umgestaltens ist ${ }^{11 "}$. Diese Situation verhindert das Entstehen einer für die Vergangenheit noch charakteristischer Beziehung zwischen Mensch und Natur. Ein bestimmtes Objekt oder die Güter der Natur werden nur genutzt. Solcher Zustand ist auch nicht gleichgültig gegenüber der Entwicklung zwischenmenschlicher Beziehungen. Für die Technik Community steht meist der „User“ im Mittelpunkt ${ }^{12}$. Der gegenwärtige technische Fortschritt zielt auf ein uneingeschränktes Wachstum ab, das aber „die Lüge bezüglich der unbegrenzten Verfügbarkeit der Güter des Planeten voraussetzt, die dazu führt, ihn bis zur Grenze und darüber hinaus auszupressen"13.

Die modernen Technologien mit ihren Methoden streben nach Kontrolle über die Wirklichkeit. „In der Tat neigt die Technik dazu — bemerkt der Papst — zu versuchen, dass nichts außerhalb ihrer harten Logik bleibt ${ }^{\text {"114 }}$. Auf diese Weise beschreibt er das Ausmaß der Realität, die die Technik abdecken möchte und betont ihre Bestrebungen, die gesamte Wirklichkeit zu erfassen. In Bezug auf Romano Guardinis Gedanken sagt der Papst ausdrücklich, dass die Technologie heute eine Lebensform ist ${ }^{15}$. Die Technologie und der technische Fortschritt sind heute Bestandteile des technokratischen Paradigmas, das die ständige Verbesserung der Lebensbedingungen verkündet. Dies gibt die Möglichkeit, das Glück für immer mehr Menschen wahr werden zu lassen. Der so verstandene technologische Fort-

${ }^{9}$ C. Miller, Technocracy and Tradition, „Communio. International Catholic Review“ 44 (2017), Nr. 4, S. 737.

${ }^{10}$ Ebd., S. 743.

${ }^{11}$ LS, Nr. 106.

${ }_{12}$ Vgl. M. Hertl, In der digitalen Subkultur, „Herder Korrespondenz“ 71 (2017), H. 6, S. 38.

13 LS, Nr. 106.

${ }^{14}$ Ebd., Nr. 108.

15 Ebd., Nr. 203. 
schritt weckt wachsende Erwartungen und weckt quasi religiöse Hoffnungen, dass er alle Umweltprobleme überwinden und letztlich kontrollieren kann ${ }^{16}$. Eine solche Sichtweise führt oft zur Verherrlichung der Technik und zur Verabsolutierung ihrer Logik, oder, wie Hans Dieter Mutschler feststellt, sogar zu einer Form der Vergöttlichung der Technik ${ }^{17}$.

Zusammenfassend lässt sich sagen, dass die päpstlichen Analysen des technischen Fortschritts deutlich auf seine Grenzen hinweisen, die durch die Eindimensionalität seiner Logik, sowie durch die reduktionistischen Annahmen der angewandten Methodik gekennzeichnet sind. Vor allem jedoch zeigt der Papst ziemlich eindeutig, dass das wissenschaftlich-technologische Paradigma nicht ausreicht, die gesamte Wirklichkeit abzudecken. Dieses einseitig technische Denken fordert das Christentum in ihrer Vision der Wirklichkeit, insbesondere in der Anthropologie heraus. Daraus ergibt sich auch seitens der Kirche das wachsende Interesse an der theologischen Reflexion über den technischen Fortschritt und seine Folgen.

Wissenschaft und Technik — sagt Franziskus — haben uns geholfen, die Grenzen der Kenntnis der Natur und insbesondere des Menschen zu vertiefen. Sie allein genügen jedoch nicht, um alle Antworten zu geben. Heute werden wir uns immer mehr bewusst, dass es notwendig ist, aus den Schätzen der Weisheit zu schöpfen, die in den religiösen Überlieferungen bewahrt werden, aus der Volksweisheit, aus der Literatur und aus den Künsten, die das Geheimnis des menschlichen Lebens in der Tiefe berühren, ohne jene zu vergessen, die in der Philosophie und in der Theologie enthalten sind. Im Gegenteil: Diese müssen neu entdeckt werden ${ }^{18}$.

\section{Bewertung des technischen Fortschritts bzw. zwischen Gut und Böse}

Die Technik selbst ist längst nicht mehr axiologisch, ethisch und sogar weltanschaulich neutral. In der bisherigen Tradition der Philosophie der Technik sind verschiedene Konzepte und Stellungnahmen zur Bewertung der Technik und Technologien entstanden. Die Bewertungen der technischen Tätigkeiten waren und sind unterschiedlich. Im Allgemeinen gibt es neben zwei extremen Haltungen, die in der Technik entweder nur das Gute oder das Böse sehen, auch eine

16 Ebd., Nr. 109.

${ }^{17}$ Vgl. H.D. Mutschler, Technik als Religionsersatz, „Scheidewege. Jahresschrift für skeptisches Denken“ 28 (1999), S. 42-54.

${ }^{18}$ Franziskus, Ansprache von Papst Franziskus an der Vollversammlung des Päpstlichen Apostolischen Rates für die Kultur (18.11.2017), [online] http://w2.vatican.va/content/francesco/de/speeches/2017/november/documents/papa-francesco_20171118_plenaria-cultura.html [12.02.2019]. 
mittlere Haltung — eine ambivalente Einschätzung. Die Befürworter für die erste Einschätzung betonen den positiven Aspekt der technologischen Entwicklung und erkennen sie als Grundlage für das menschliche Wohlbefinden und als eine Quelle der maximalen Befriedigung der menschlichen Bedürfnisse. Die Probleme mit negativen Auswirkungen der technologischen Entwicklung werden in dieser Hinsicht marginalisiert oder ganz ignoriert. Auf der anderen Seite betonen die Vertreter die entgegengesetzten Position nur die negativen Auswirkungen der technischen Entwicklung und weisen auf die Gefahr einer totalen Atom- oder Umweltkatastrophe hin, die zum Aussterben allen Lebens auf der Erde führen kann. Heutzutage beinhaltet die ethische Bewertung immer häufiger Meinungen, dass der Fortschritt zu guten und zu schlechten Ergebnissen führen kann. Eine solche Mehrdeutigkeit in der Bewertung zeigt sich zumindest in den unterschiedlichen Antworten auf die Frage: Ist alles, was technisch möglich ist, auch ethisch zulässig? ${ }^{19}$

Die Ambivalenz bei der Bewertung technologischer Leistungen zeigt sich auch bei Papst Franziskus. Angefangen mit einer Erinnerung an die positiven Bewertungen des technischen Fortschritts seiner Vorgänger, weist der Papst auf den Beitrag des wissenschaftlichen und technischen Fortschritts insbesondere in den Bereichen der Medizin, Technik und Kommunikation zum Wohl der ganzen Menschheit hin ${ }^{20}$. Er betont nicht nur messbare Vorteile, sondern auch einige metaphysische Funktionen des Fortschritts, die sich mit der Öffnung des Menschen für die Schönheit verbinden lassen. „Die gut ausgerichtete Technoscience — äuBert sich der Papst - ist ebenso in der Lage, das Schöne hervorzubringen und den in die materielle Welt eingetauchten Menschen in die Sphäre der Schönheit «springen» zu lassen. [...] So vollzieht sich bei der Suche des technischen Erzeugers nach Schönheit und im Betrachter dieser Schönheit ein Sprung in eine gewisse echt menschliche Fülle ${ }^{21 ،}$. Trotz positiver Worte über den Beitrag des technischen Fortschritts zur Entwicklung der Menschheit, und das nicht nur in ihrer materiellen Hinsicht, betrachtet der Papst die Technologie nicht als bedingungslosen Segen. Neben vielen messbaren Vorteilen dank des Einsatzes neuer Technologien übersieht der Papst auch nicht einige Risiken, die mit ihrer Nutzung der neuen Technologien verbunden sind. Sie führen nicht selten zur Herrschaft einer Gesellschaft über eine andere oder sogar zur Vernichtung der Menschheit, wie dies beim Einsatz der Atombombe der Fall war ${ }^{22}$. Seine klare Kritik orientiert sich vor allem auf die besitzorientierte Technik, die sich nicht nur mit die Kont-

${ }^{19}$ Vgl. A. Bogner, Einführung: Zur Ethisierung der Technik [in:] Ethisierung der Technik..., S. 9-10.

${ }^{20} \mathrm{Vgl}$. LS, Nr. 102.

${ }^{21}$ Ebd., Nr. 103; Vgl. auch A. Ganowicz-Bączyk, Wątki ekoetyczne w encyklice Laudato si' papieża Franciszka [in:] Kościót i nauka w obliczu ekologicznych wyzwań, red. J. Poznański, S. Jaromi, Kraków 2016, S. 208-209.

${ }^{22}$ Vgl. LS, Nr. 104. 
rolle der Natur abgibt, sondern auch die Herrschaft über die sozialen, wirtschaftlichen oder politischen Dimensionen zu gewinnen anstrebt ${ }^{23}$. Was schließlich für die Technik zählt, ist der Gewinn, unabhängig von den Folgen der Zerstörung der Natur oder der Verarmung ganzer Gesellschaften durch ihre Ausgrenzung ${ }^{24}$. Die technischen Produkte sind nicht neutral, „,denn sie schaffen ein Netz, das schließlich die Lebensstile konditioniert und lenken die sozialen Möglichkeiten in die

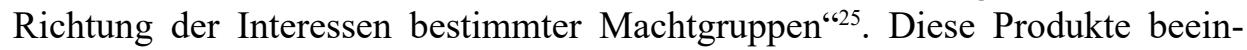
flussen stark den Lebensstil und oft bestimmen sie in Übereinstimmung mit den Interessen bestimmter Machtgruppen die Entwicklungsrichtungen der einzelnen Gesellschaften ${ }^{26}$. Deshalb ruft der Papst zum Widerstand gegen die Erweiterung des technokratischen Paradigmas auf ${ }^{27}$.

Die ambivalente Bewertung des technischen Fortschritts ist nicht das letzte Wort der päpstlichen Reflexion über dieses gegenwärtige Zeichen der Zeit, nämlich das Phänomen des dynamischen technischen Fortschritts. Franziskus versucht auch einige konkrete Postulate über eine neue Idee des wissenschaftlichtechnischen Fortschritts zu formulieren. Es sind Postulate, die für eine integrale Sicht der Wirklichkeit offen zu sein scheinen.

\section{Zu integraler Sicht der Wirklichkeit bzw. zwischen Teil und Ganzem}

Auf der Suche nach einem ganzheitlichen Zugang zur Wirklichkeit besteht nicht nur kein Widerspruch zwischen dem christlichen Glauben und dem technologischen Fortschritt, sondern es wird das immer positiver das größer werdende Bewusstsein über die klareren Verbindungen zwischen verschiedenen Aspekten der Wirklichkeit gesehen. „In der Welt - lesen wir in der Einführung zur Laudato si' - ist alles miteinander verbunden; die Kritik am neuen Machtmodell und den Formen der Macht, die aus der Technik abgeleitet sind; die Einladung, nach einem anderen Verständnis von Wirtschaft und Fortschritt zu suchen“"28. Die Anerkennung der in der Realität vorhandenen komplexen Zusammenhänge ist eine klare Herausforderung auf der Suche nach einer ganzheitlichen Sicht der Wirklichkeit, so wie auch eine Notwendigkeit, eine neue Lebensweise zu entwickeln. Nur eine solche Auffassung der Wirklichkeit gibt die Möglichkeit, ihre

${ }^{23}$ Ebd., Nr. 107, 109.

${ }^{24}$ Vgl. Th. Wieland, Laudato si’ praktisch, „Herder Korrespondenz“ 70 (2016), Nr. 11, S. 49.

${ }^{25}$ LS, Nr. 107.

${ }^{26}$ Vgl. J. Bremer, Encyklika Laudato si' - ekologia integralna podstawowym elementem katolickiej nauki społecznej [in:] Kościót i nauka w obliczu ekologicznych wyzwań, red. J. Poznański, S. Jaromi, Kraków 2016, S. 41-42.

${ }^{27}$ LS, Nr. 111.

${ }^{28}$ Ebd., Nr. 16. 
verschiedenen Dimensionen zu integrieren und die Krise, die mit dem Verlust der Bedeutung des Ganzen verbunden ist, zu überwinden.

Die der Technologie eigene Spezialisierung — schreibt der Papst — bringt eine große Schwierigkeit mit sich, das Ganze in den Blick zu nehmen. Die Aufsplitterung des Wissens erfüllt ihre Funktion, wenn sie konkrete Anwendungen erzielt, führt aber gewöhnlich dazu, den Sinn für die Gesamtheit, für die zwischen den Dingen bestehenden Beziehungen, für den weiten Horizont zu verlieren, der irrelevant wird ${ }^{29}$.

Die für den wissenschaftlich-technischer Fortschritt spezifische Spezialisierung führt dazu, dass das Wissen, das eine Person erreicht, fragmentarisch ist und „aus diesem Grund können die bruchstückhaften und isolierten Kenntnisse zu einer Art von Ignoranz werden, wenn sie sich nicht in eine umfassendere Sicht der Wirklichkeit einfügen lassen“"30. Diese Art von Wissen bietet keine Möglichkeit, die komplexen Probleme der Menschheit zu lösen, insbesondere solche, die sich auf ökologische oder soziale Fragen beziehen. Die Eindimensionalität des wissenschaftlich-technischen Fortschritts deckt nicht die gesamte Wirklichkeit ab. Deswegen plädiert der Papst für die Herausarbeitung einer neuen Idee der technologischen Fortschritts. Diese soll sich nicht nur an den Zahlen und den materiellen Maßstäben der Produktivität orientieren, sondern auch für eine Verbesserung der Lebensqualität und eine mehr integrierte Welt einen ganz entscheidenden Beitrag leisten. Es sei darauf hingewiesen, dass die vorgeschlagene Idee nicht nur die theoretischen Bemerkungen über die Wechselwirkung zwischen der technischen Vernunft und einer für die Transzendenz offenen Vernunft enthält, sondern sie bezieht sich auch sich auf einige praktische Handlungen, die von der christlichen Offenbarung inspiriert sind. In Grunde genommen geht es dem Papst um eine Harmonie zwischen dem technischen Fortschritt und anderen Dimensionen der Wirklichkeit.

Welche Haltungen sind mit der integralen Sicht der Wirklichkeit verbunden? Welche konkrete praktischen Handlungen schlägt der Papst vor? Es scheint, dass es in erster Linie um einen kritischen Aufsatz gegenüber den reduktionistischen Tendenzen des technischen Fortschritts geht. Der Papst zeigt die Gefahren einer Forschung, die sich nur auf quantifizierbare und formale Aspekte der Wirklichkeit beschränkt. Die Berücksichtigung der Wirklichkeit nur im Hinblick auf die technische Vernunft führt vor allem zur Entfremdung des Menschen von der Bedeutung der transzendentalen Qualitäten und zur Reduzierung der zwischenmenschlichen Beziehungen zu völlig formalisierten Verhältnissen. Eine kritische

\footnotetext{
29 Ebd., Nr. 110.

${ }^{30}$ Ebd., Nr. 138.
} 
Haltung drückt sich auch darin aus, die Gefahren, die mit der Verformung des Sinnes und der Perspektiven des menschlichen Lebens verbunden sind, aufzudecken. Die Einschränkung der Wirklichkeit auf das enge Verständnis der Wissenschaft ist der Grund dieser Reduzierung des Gesamtsinns des Lebens. Diese Kritik richtet sich gegen jedem Versuch der Verabsolutierung der wissenschaftlich-technischen Forschungsperspektive sowie gegen die Technisierung der gesamten Kultur. Die kritische Haltung des Papstes gegenüber der Eindimensionalität des technologischen Fortschritts bedeutet nicht eine Infragestellung als solche, sondern der Papst verlangt eine klare Anerkennung seiner Grenzen in Bezug auf die Breite der Kenntnis der Wirklichkeit und die Fähigkeit, die menschlichen Bedürfnisse zu befriedigen ${ }^{31}$.

Die Gefahr einer „methodischen“ Verarmung der Wirklichkeit durch Beschränkung auf den technischen Fortschritt kann nur im Dialog mit anderen wissenschaftlichen Disziplinen überwunden werden. Der Papst ruft dazu auf, „die tragische Spaltung zwischen den 'zwei Kulturen' der humanistisch-literarisch-theologischen und der naturwissenschaftlichen - die zu einer gegenseitigen Verarmung führt, zu überwinden und einen umfassenderen Dialog auch zwischen Kirche, der Gemeinschaft der Gläubigen und der Wissenschaftsgemeinschaft zu ermutigen“32. Daher ist die Suche nach dem integralen Zugang zur Wirklichkeit auch durch die Forderung nach einer Öffnung der Technologie für den Dialog mit Philosophie und Theologie gekennzeichnet ${ }^{33}$. Ein solcher Dialog scheint ein wichtiger Faktor für die Verwirklichung einer neuen Idee des technologischen Fortschritts zu sein. Der Dialog ermöglicht interdisziplinäre Aktivitäten, die auf ein umfassenderes Verständnis der Wahrheit über den Menschen, der Welt um ihn herum und der Beziehungen zwischen ihnen abzielen. Dieser Dialog gibt außerdem mit den verschiedenen Bereichen der Geisteswissenschaften der Technologie die Möglichkeit, die ganzheitliche Sicht der Wirklichkeit nicht zu verlieren und die vielfältigen Beziehungen zwischen den verschiedenen Dimensionen zu harmonisieren ${ }^{34}$. Ein solcher Dialog trägt zur Entwicklung eines neuen Denkens und globalen Handelns bei, wobei die Interessen künftiger Generationen berücksichtigt werden. Es geht um ein Denken, das alle menschlichen Bedürfnisse und Werte, einschließlich der ästhetischen, kulturellen und religiösen umfasst. Ein fruchtbarer Dialog kann sich positiv auf die Entstehung einer neuen Kultur im Umgang mit der Technik und ihrer Produkte auswirken. Diese Art des Denkens kann bei der Integration des technischen und religiösen Wissens helfen. Eine

${ }^{31}$ Vgl. ebd., Nr. 107, 109.

32 Franziskus, Ansprache...

33 LS, Nr. 60.

${ }^{34}$ Vgl. A. Ganowicz-Bączyk, Wątki ekoetyczne..., S. 207; J. Bremer, Encyklika Laudato si’..., S. $39-40$. 
Synthese der beiden unterschiedlichen Arten von Wissen ist für die Einzelperson von größter Bedeutung, die als VertreterInnen der wissenschaftlich-technischen Zivilisation oder als aktive VertreterInnen der technischen Wissenschaften und Mitgestalter des technologischen Fortschritts gleichzeitig zur Erlösung berufen sind.

Der Weg zur ganzheitlichen Sicht der Wirklichkeit kann auch nicht einige praktische Haltungen als Ausdruck der christlichen Orthopraxie, wie z. B. Verantwortung, Solidarität, Selbstbeschränkung oder Askese überspringen. Die Verantwortung für den technischen Fortschritt ist nicht nur eine Domäne der Kirche und ihrer Theologie ${ }^{35}$. Die Analyse der Umweltkrise zeigt, dass sie nicht nur eine natürliche und technische Dimension hat, sondern eng mit der moralischen Krise verbunden ist. „Die Verschlechterung der Umweltbedingungen und die Verschlechterung im menschlichen und ethischen Bereich sind - wie der Papst bemerkt - im menschlichen und ethischen Bereich eng miteinander verbunden"36. Die unangemessene menschliche Aktivität in Bezug auf die natürliche oder soziale Umwelt ist oft auf philosophische, religiöse, ethische und wirtschaftliche Überzeugungen zurückzuführen. Die Krise der moralischen Werte des Menschen, denen die materiellen Werte vor den persönlichen Werten vorgezogen werden, führt zu einer unverantwortlichen Ausbeutung der natürlichen Welt. Die spezifischen negativen Auswirkungen menschlichen Handelns in einer von ethischen Kriterien befreiten Welt werden von Papst Franziskus am Beispiel der Analyse von Phänomenen wie Umweltverschmutzung und globaler Erwärmung, Wassermangel, Verlust der biologischen Vielfalt, Verschlechterung der Lebensqualität oder der sozialen Ungerechtigkeit ausführlich diskutiert ${ }^{37}$. Deshalb verlangt der Papst die Übernahme und Vertiefung eines Gefühls der moralischen Verantwortung. Präzise spricht er von unterschiedlichen Formen der Verantwortlichkeit, für andere Menschen und die soziale Umwelt ${ }^{38}$, für zukünftige Generationen $^{39}$, für Naturgüter ${ }^{40}$, so wie für die moralische Entwicklung des Menschen im Verhältnis zur Entwicklung der Zivilisation.

Wir müssen wieder spüren — appelliert er — dass wir einander brauchen, dass wir eine Verantwortung für die anderen und für die Welt haben und dass es sich lohnt, gut und ehrlich zu sein. Wir haben schon sehr viel Zeit moralischen Verfalls verstreichen lassen, indem wir die Ethik, die Güte, den Glauben und die Ehrlichkeit

35 Vgl. Ch. Hubig, Technik [in:] Lexikon der Wirtschaftsethik, hrsg. v. G. Enderle, Freiburg 1993, S. 1079-1081.

${ }^{36}$ LS, Nr. 56.

37 Vgl. ebd., Nr. 17-59.

${ }^{38}$ Vgl. ebd., Nr. 25, 45, 90.

39 Vgl. ebd., Nr. 109, 165.

${ }^{40}$ Vgl. ebd., Nr. 59, 68, 78, 211. 
bespöttelt haben, und es ist der Moment gekommen zu merken, dass diese fröhliche Oberflächlichkeit uns wenig genützt hat ${ }^{41}$.

Diese Situation macht es notwendig, die entsprechenden Maßnahmen zu ergreifen, um die Folgen zu überwinden und eine Umweltkatastrophe zu vermeiden. Es ist wichtig, neben den bereits ergriffenen rechtlichen und politischen Initiativen zum Schutz der Natur vor weiteren Zerstörungen und zur Regeneration, alle Maßnahmen zu ergreifen, die den Schutz der Natur auch auf ethischer und moralischer Ebene fördern. Der Mensch als moralisches Wesen sollte sich der moralischen Verantwortung für die ihn umgebende Wirklichkeit bewusst sein. Es geht darum, das richtige Gleichgewicht zwischen der zivilisatorischen Entwicklung der Menschheit und der moralischen Entwicklung zu bewahren ${ }^{42}$. In der Verbindung dieser beiden Themen kann man den praktischen Beitrag der Theologie und der christlichen Kultur zur Humanisierung der Technik im Bereich der menschlichen Bildung sehen. Dieser Beitrag scheint, im Vergleich zur menschlichen Bildung, schwieriger umzusetzen zu sein, aber es ist ein wichtiges Element in der Sorge um die ganzheitliche Entwicklung des Menschen.

Der technologische Fortschritt betrifft nicht nur die Umwelt, sondern auch den sozialen Fortschritt. In dem letzteren werden Menschen und ganze Gesellschaften durch globale Ungerechtigkeiten degradiert. Daher kann das Postulat eines integralen Wirklichkeitszugangs die Werte, die ein Gegenmittel zum Problem der sozialen Ungerechtigkeit darstellen können, nicht ignorieren. Der Papst räumt hier der Haltung der Solidarität einen besonderen Platz ein. Er betont besonders die Solidarität zwischen den Generationen. Ohne diese Art von Solidarität

kann von nachhaltiger Entwicklung keine Rede mehr sein. Wenn wir an die Situation denken, in der der Planet den kommenden Generationen hinterlassen wird, treten wir in eine andere Logik ein, in die des freien Geschenks, das wir empfangen und weitergeben. Wenn die Erde uns geschenkt ist, dann können wir nicht mehr von einem utilitaristischen Kriterium der Effizienz und der Produktivität für den individuellen Nutzen her denken ${ }^{43}$.

Die Beibehaltung solcher Haltung ist grundsätzlich unmöglich, ohne eine evangelische Haltung der Askese gegenüber der Gütern des technischen Fortschritts aufzubauen. Franziskus spricht hier über eine Ethik der Selbstbeschränkung und

${ }^{41}$ Ebd., Nr. 127.

${ }^{42}$ A. Dylus, Integralna ekologia papieża Franciszka. Perspektywa etyki odpowiedzialności [in:] Świat jako wspólny dom. Wokót koncepcji ekologii integralnej w encyklice Laudato si', red. A. Wysocki, Warszawa 2016, S. 126.

${ }^{43}$ LS, Nr. 159; siehe auch Nr. 162, 172. 
versucht zu zeigen, dass es hinter dem politischen Diskurs über die Macht der Technik einen anthropologischen gibt. Dieser führt direkt zum Kern der Gedanken des Papstes. „Es gibt keine Ökologie ohne angemessene Anthropologie ${ }^{\text {“44 }}$. Es ist gut und richtig, den Nutzen des technischen Fortschrittes für eine nachhaltige Entwicklung zu schätzen und anzuerkennen. Die Technologien ,geben denen, welche die Kenntnis und vor allem die wirtschaftliche Macht besitzen, sie einzusetzen, eine beeindruckende Gewalt über die gesamte Menschheit und die ganze Welt ${ }^{\text {"455 }}$. Deswegen brauche die Menschheit eine solide Ethik, eine Kultur und eine Spiritualität, die Grenzen setzen und die Selbstbeschränkung lehren ${ }^{46}$. Es geht um ein größeres Bewusstsein, besonders unter den Christen, für die Notwendigkeit, Mensch und Welt als Werk Gottes zu betrachten. Die Motivation des Menschen, sich um das Schöpfungswerk zum Wohl zukünftiger Generationen zu kümmern, sollte sich daher nicht nur auf humanistische Gründe beziehen, sondern auch auf die christliche Offenbarung. Der praktische Beitrag der Theologie, insbesondere der Theologie der Spiritualität, besteht darin, die Askese des Menschen in der Anwendung technischer Errungenschaften zu lehren. Eine solche Haltung kann immer als eine Antwort aus der Perspektive der christlichen Anthropologie auf die spezifischen negativen Auswirkungen der technischen Zivilisation, wie z.B.: übermäßiger Konsum, Abfall, Umweltverschmutzung, Rücksichtslosigkeit und Raub von Rohstoffen, verstanden werden. Um eine solche Haltung zu entwickeln, ist nach Franziskus eine ökologische Umkehr dringend nötig. Dank dieser Form der Bekehrung kann eine asketische Haltung gebildet werden. Sie drückt sich konkret in der Gewohnheit aus, zu sparen, der Selbstbeherrschung und bildet sie so eine wirksame Begrenzung der Auswirkungen von überschwänglichem Individualismus, Egoismus, Hedonismus oder Konsumismus ${ }^{47}$.

$* * *$

Das Spektrum der Reaktionen auf den technischen Fortschritt erstreckt sich heute von radikal optimistischen Positionen bis zu skeptischen oder sogar extrem negativen aus. Zu diesen Bewertungen gehört auch die Stimme der Kirche, die sich in der päpstlichen Lehre ausdrückt, die den positiven Beitrag des wissenschaftlichtechnischen Fortschritts zur Entwicklung der menschlichen Kultur, des würdigen Lebens und des sozialen Wachstums eindeutig anerkennt. Gleichzeitig blendet die kirchliche kritische Würdigung jedoch nicht die Bedrohungen seitens der Technologie aus, die sich insbesondere im Bereich der Umwelt- und Sozialfragen

${ }^{44}$ Ebd., Nr. 118.

45 Ebd., Nr. 104.

46 Ebd., Nr. 105.

${ }^{47}$ Vgl. A. Ganowicz-Bączyk, Wątki ekoetyczne..., S. 208; M. Tatar, Ontyczna duchowość stworzenia w perspektywie Laudato si' [in:] Świat jako wspólny dom. Wokót koncepcji ekologii integralnej w encyklice Laudato si', red. A. Wysocki, Warszawa 2016, S. 75-77. 
zeigen. Die Kirche unterstützt Technologien, die gute moralische Handlungen ermöglichen und lehnt diejenigen, die zu schlechten moralischen Handlungen führen, ab.

Worin drückt sich die Originalität des Papstes in seiner kritischen Würdigung des technischen Fortschritts aus? Was kennzeichnet seinen Vorschlag für eine neue Fortschrittsidee? Zusammenfassend kann man sagen, dass Franziskus eine klare Unterscheidung zwischen guten, neutralen und schlechten Technologien trifft. Der entscheidende Faktor für die ethische Bewertung des wissenschaftlich-technischen Fortschritts ist, ob er gute oder schlechte moralische Handlungen ermöglicht. Seine Kritik richtet sich nicht gegen die Technologie als solche, sondern nur gegen alle unkontrollierten Formen des wissenschaftlich-technischen Fortschritts, die dem Schöpfungswerk und dem menschlichen Leben schaden. Diese Kritik ist besonders deutlich, wenn dieser Fortschritt mit einer Machterhöhung und einer Herrschaft über immer größere Bereiche der Wirklichkeit verbunden ist. Er kritisiert eindeutig den Fortschritt überall dort, wo nur seine Effizienz, unmittelbare wirtschaftliche Vorteile und seine Unabhängigkeit von irgendwelchen ethischen Bezügen berücksichtigt werden. Solches technokratische Paradigma verletzt zweifellos die katholische Tradition der Interaktion zwischen christlicher Ethik und Moral und wissenschaftlich-technischem Fortschritt. Daher verlangt der Papst, noch stärker als seine Vorgänger, die Reflexion über den wissenschaftlich-technischen Fortschritt im Kontext seiner Verknüpfungen mit den anderen Dimensionen der Wirklichkeit zu führen. Es scheint sein Verdienst zu sein, dass er eindeutig den Dialog zwischen den Vertretern der Technik und den Philosophen und Theologen befürwortet. Das Wesen der neuen Idee des technischen Fortschritts besteht darin, zu erkennen, dass in der Wirklichkeit alles miteinander verbunden ist. Solches Bewusstsein für die bestehenden Verbindungen fördert die Wiederherstellung der ursprünglichen Einheit zwischen Homo faber, Homo sapiens und Homo religiosus. Der Aufbau einer solchen Einheit fördert noch mehr eine Humanisierung des Fortschritts mit den Werten des Evangeliums Christi.

Die Evangelisierung — schreibt er — achtet auf die wissenschaftlichen Fortschritte, um sie mit dem Licht des Glaubens und des Naturrechts zu erleuchten, damit sie immer die Zentralität und den höchsten Wert des Menschen in allen Phasen seines Lebens respektieren. Die gesamte Gesellschaft kann bereichert werden dank diesem Dialog, der dem Denken neue Horizonte öffnet und die Möglichkeiten der Vernunft erweitert. Auch das ist ein Weg der Harmonie und der Befriedung ${ }^{48}$.

${ }^{48}$ Franziskus, Apostolisches Schreiben Evangelii Gaudium (2013), Nr. 243. 
Der Papst bemüht sich um den Aufbau einer ganzheitlichen Sicht der Wirklichkeit, in der der technische Fortschritt nicht der einzige Akteur ist. Das bedeutet aus theologischer Sicht, den wissenschaftlich-technischen Fortschritt mit der Ethik und der katholischen Soziallehre zu verbinden. Die neue Idee des Fortschritts kann die konkreten praktischen Auswirkungen, die der Papst mit dem Stichwort „ökologische Konversion“ bezeichnet, nicht ausweichen. Das heißt, es müssen konkrete Handlungen erfüllt werden, um ein integrales Konzept der Wirklichkeit zu realisieren. Darüber hinaus kann man in den vom Papst klar formulierten Vorschlägen eine Gründung systemischer Grundlagen sehen, die von der theologischen Perspektive inspiriert sind. Es geht um langfristige Maßnahmen, die auf eine ganzheitliche Sicht der Wirklichkeit abzielen und zwar nicht unverzüglich angesichts von Katastrophen, die durch die technologische Entwicklung verursacht werden.

Die päpstlichen Impulse für eine neue Idee des wissenschaftlich-technischen Fortschritts bilden einen wichtigen Beitrag zum Aufbau einer Theologie der Technik, die heute mehr als in der Vergangenheit ein Imperativ scheint, der von der Schöpfungstheologie und christlichen Anthropologie inspiriert ist. Der Klimawandel, die angesammelten Kernwaffen, eine unkontrollierte Entwicklung der Nanotechnologie und die künstliche Intelligenz können in Zukunft eine reale Bedrohung für die menschliche Existenz darstellen. Deswegen ist eine der wesentlichen Aufgaben der Kirche in der irdischen Ordnung die Sorge um das Schöpfungswerk und den Menschen selbst. Manchmal hat die Kirche sogar die Verteidigung der Menschheit gegen Bedrohungen des befreiten von externen Referenzen wissenschaftlich-technischem Fortschritts vorzunehmen. So scheint die Kooperation zwischen Rom und Silicon Valley mehr als eine Notwendigkeit zu sein, nicht nur, um unser gemeinsames Haus zu schützen, sondern es auch harmonisch miteinander aufzubauen.

\section{Between Rome and Silicon Valley. Pope Francis in the face of technical progress}

\section{Summary}

What has Rome to do with Silicon Valley? In other words, what has the Church today to do with science and technology? Does the Church have any interest in a debate with the present scientific and technical progress, of which Silicon Valley is a symbol? The aim of my research is to look for a new idea of technological progress that will allow to build an integral vision of reality. The teaching of Pope Francis, especially in the Encyclical 'Laudato si'(2015), provides the basis for the undertaken topic. The structure of the considerations refers to the principles of the social doctrine of the so-called three steps: look, judge, act. Therefore, in the course of the considerations firstly 
the features of technical progress will be presented (1), then its evaluation (2), and finally elements of an integral vision of reality (3).

\section{Keywords}

Pope Francis, theology, technical progress, integral vision of reality

\section{Pomiędzy Rzymem a Silicon Valley. Papież Franciszek wobec postępu technicznego}

\section{Streszczenie}

Co Rzym ma wspólnego z Doliną Krzemową? Innymi słowy, co Kościół ma współcześnie wspólnego z nauką i technologią? Jak Kościół odnosi się do problematyki postępu naukowo-technicznego, którego symbolem jest Dolina Krzemowa? Celem podjętych rozważań jest poszukiwanie nowej koncepcji postępu technicznego, uwzględniającej całościową wizję rzeczywistości. Nauczanie papieża Franciszka, zwłaszcza z Encykliki Laudato si' (2015), stanowi podstawę realizacji wybranego tematu. Struktura rozważań nawiązuje do zasad nauki społecznej tzw. trzech kroków: zbadać, ocenić, działać. Dlatego w trakcie rozważań są prezentowane najpierw cechy postępu technicznego (1), dokonana jego ocena (2), a następnie elementy integralnej wizji rzeczywistości (3).

\section{Słowa kluczowe}

papież Franciszek, teologia, postęp techniczny, integralna wizja rzeczywistości

\section{Literaturverzeichnis}

Bogner A., Einführung: Zur Ethisierung der Technik [in:] Ethisierung der Technik Technisierung der Ethik. Der Ethik-Boom im Lichte der Wissenschafts- und Technikforschung, hrsg. v. A. Bogner, Baden-Baden 2013, s. 7-25.

Bremer J., Encyklika Laudato si' - ekologia integralna podstawowym elementem katolickiej nauki społecznej [in:] Kościót i nauka w obliczu ekologicznych wyzwań, red. J. Poznański, S. Jaromi, Kraków 2016, S. 37-54.

Dylus A., Integralna ekologia papieża Franciszka. Perspektywa etyki odpowiedzialności [in:] Świat jako wspólny dom. Wokót koncepcji ekologii integralnej w encyklice Laudato si', red. A. Wysocki, Warszawa 2016, S. 109-129.

Edenhofer O., Klima, Kohle, Kapital. Zum Stand der internationalen Klimapolitik, „Stimmen der Zeit“" 143 (2018) 4, S. 255-264.

Endras A., Die Welt brennt, der Gipfel streitet, „Zeit Online“, https://www.zeit.de/wirtschaft/2018-12/un-klimakonferenz-welt-klimagipfel-klimaschutz-kattowitz [Abruf: 25.01.2019]. 
Ethisierung der Technik - Technisierung der Ethik. Der Ethik-Boom im Lichte der Wissenschafts- und Technikforschung, hrsg. v. A. Bogner, Baden-Baden 2013.

Franziskus, Ansprache von Papst Franziskus an der Vollversammlung des Päpstlichen Apostolischen Rates für die Kultur (18.11.2017), http://w2.vatican.va/content/francesco/de/speeches/2017/november/documents/papa-francesco_20171118_plenariacultura.html [Abruf: 12.02.2019].

Franziskus, Apostolisches Schreiben Evangelii Gaudium (2013), http://w2.vatican.va/ content/francesco/de/apost_exhortations/documents/papa-francesco_esortazioneap_20131124_evangelii-gaudium.html [Abruf: 15.01.2019].

Franziskus, Enzyklika Laudato si' (2015), https://www.katholisch.at/laudatosi [Abruf: 16.02.2019].

Ganowicz-Bączyk A., Watki ekoetyczne w encyklice Laudato si’ papieża Franciszka [in:] Kościół i nauka w obliczu ekologicznych wyzwań, red. J. Poznański, S. Jaromi, Kraków 2016, S. 193-214.

Hertl M., In der digitalen Subkultur, „Herder Korrespondenz“ 71 (2017) 6, S. 37-39.

Hubig Ch., Technik [in:] Lexikon der Wirtschaftsethik, hrsg. v. G. Enderle, Freiburg 1993, S. 1079-1081.

Johannes XXIII, Enzyklika Mater et Magistra (1961), http://w2.vatican.va/content/ john-xxiii/de/encyclicals/documents/hf_j-xxiii_enc_15051961_mater.html [Abruf: 16.02.2019].

Miller C., Technocracy and Tradition, „Communio. International Catholic Review“ 44 (2017) 4, S. 718-746.

Mutschler H.D., Die Gottmaschine. Das Schicksal Gottes im Zeitalter der Technik, Augsburg 1998.

Mutschler H.D., Technik als Religionsersatz, „Scheidewege. Jahresschrift für skeptisches Denken“ 28 (1999), S. 42-54.

Nordmann A., Technikphilosophie zur Einführung, Hamburg 2015.

Schleissing S., Laien, Experten, Propheten: Zur Rolle der Theologen in Technikdiskursen [in:] Technik und Lebenswirklichkeit: Philosophische und theologische Deutungen der Technik im Zeitalter der Moderne, hrsg. v. A.M. Richter, Ch. Schwarke, Stuttgart 2014, S. 203-216.

Tatar M., Ontyczna duchowość stworzenia w perspektywie Laudato si' [in:] Świat jako wspólny dom. Wokót koncepcji ekologii integralnej $w$ encyklice Laudato si', red. A. Wysocki, Warszawa 2016, S. 61-82.

Wieland Th., Laudato si’ praktisch, „Herder Korrespondenz“ 70 (2016), Nr. 11, S. 47-50. 